\title{
De-noising of Underwater Acoustic Signals Based on ICA Feature Extraction
}

\author{
Kong Wei and Yang Bin \\ Information Engineering College, Shanghai Maritime University, Shanghai 200135, China \\ kongwei@sjtu.edu.cn, binyang@cie.shmtu.edu.cn
}

\begin{abstract}
As an efficient sparse coding and feature extraction method, independent component analysis (ICA) has been widely used in speech signal processing. In this paper, ICA method is studied in extracting low frequency features of underwater acoustic signals. The generalized Gaussian model (GGM) is introduced as the p.d.f. estimator in ICA to extract the basis vectors. It is demonstrated that the ICA features of ship radiated signals are localized both in time and frequency domain. Based on the ICA features, an extended denoising method is proposed for underwater acoustic signals which can extract the basis vectors directly from the noisy observation. The de-noising experiments of underwater acoustic signals show that the proposed method offers an efficient approach for detecting weak underwater acoustic signals from noise environment.
\end{abstract}

\section{Introduction}

Recently, independent component analysis (ICA) has been shown highly effective in encoding patterns, including image and speech signals ${ }^{[1-4]}$. Unlike correlation-based learning algorithm, ICA can extract the higher order statistics from data. And the most informative features of sound signals require higher-order statistics for their characterization. Nowadays, pattern recognition and object detection of underwater acoustic signals are hard works since these signals are non-Gaussian, non-stationary and non-linear complex signals. In this paper, ICA is used in extracting the higherorder statistics of underwater acoustic signals, and the generalized Gaussian model (GGM) was introduced in ICA algorithm to estimate the p.d.f. of coefficients. By inferring only one parameter $q$, ICA algorithm can extract the efficient basis vectors for different underwater acoustic signals. The time and frequency domain characteristic of the ICA basis and the sparseness of coefficients demonstrate that ICA feature extraction of underwater acoustic signals is efficient.

Based on the ICA features, an extended de-noising method is proposed for noisy underwater acoustic signals. In many ICA-based de-noising works, the de-noising process of noisy signals needs noise-free source data to train the ICA basis vectors as a priori knowledge. Unfortunately, the noise-free signal is always not acquirable in practice. In this paper, the ICA algorithm based on GGM is presented on extracting the efficient basis vectors directly from the noisy signals. At the same time the shrinkage function can be obtained from the p.d.f. of each coefficient. Using the maximum likelihood (ML) method on the non-Gaussian variables corrupted by 
additive white Gaussian noise, we show how to apply the shrinkage method on the coefficients to reduce noise. The de-noising experiments of the artificial mixtures of underwater acoustic signals show that the short-term zero crossing rate (ZCR) of source signals is improved after de-noising.

\section{ICA Feature Extraction Using GGM}

ICA assume the observation $\boldsymbol{x}$ is the linear mixture of the independent components $\boldsymbol{u}$, $\boldsymbol{x}=\boldsymbol{A s}$, where the columns of $\boldsymbol{A}$ are described as the basis vectors. An ICA feature extraction algorithm is applied to obtain independent vectors $\boldsymbol{u}$ and weight matrix $\boldsymbol{W}$ from signal segment $\boldsymbol{x}, \boldsymbol{u}=\boldsymbol{W} \boldsymbol{x}$, then the basis vectors $\boldsymbol{A}$ can be calculated by the relation $\boldsymbol{A}=\boldsymbol{W}^{-1}$. The infomax learning rule is used here ${ }^{[1-4]}$ :

$$
\Delta W \propto \eta\left[I-\varphi(s) s^{T}\right] W
$$

Where the vector $\varphi(s)$ is a function of the prior and is defined by $\varphi(s)=-\frac{\partial \log p(s)}{\partial s}$, here $p(s)$ are the p.d.f.s of vectors $s$. It can be seen that the knowledge of the p.d.f. of the independent components $s$ plays an important role. Here the generalized Gaussian model (GGM) is used in ICA feature extraction of underwater acoustic signals.

The GGM models a family of density functions that is peaked and symmetric at the mean, with a varying degree of normality in the following general form ${ }^{[5,6]}$

$$
p_{g}(s \mid \theta)=\frac{\omega(q)}{\sigma} \exp \left[-c(q)\left|\frac{s-\mu}{\sigma}\right|^{q}\right], \quad \theta=\{\mu, \theta, q\}
$$

where

$$
c(q)=\left[\frac{\Gamma[3 / q]}{\Gamma[1 / q]}\right]^{q / 2}, \quad \omega(q)=\frac{\Gamma[3 / q]^{1 / 2}}{(2 / q) \Gamma[3 / q]^{3 / 2}}
$$

$\mu=E[s]$ and $\sigma=\sqrt{E\left[(s-\mu)^{2}\right]}$ are the mean and standard deviation of the data respectively. $\Gamma[\cdot]$ is the Gamma function. By inferring $q$, a wide class of statistical distributions can be characterized. The Gaussian, Laplacian, and strong Laplacian distributions can be modeled by putting $q=2, q=1$, and $q<1$ respectively. In ICA learning rules, the problem then becomes to estimate the value of $q$ from the data. This can be accomplished by simply finding the maximum posteriori value $q$. The posterior distribution of $q$ given the observations $\boldsymbol{x}=\left\{x_{1}, \ldots, x_{\mathrm{n}}\right\}$ is

$$
p(q \mid x) \propto p(x \mid q) p(q)
$$

where the data likelihood is

$$
p(x \mid q)=\prod_{n} \omega(q) \exp \left[-c(q)\left|x_{n}\right|^{q}\right]
$$


and $p(q)$ defines the prior distribution for $q$. Gamma function $\Gamma[\cdot]$ is used as $p(q)$ here.

In the case of the GGM as the p.d.f of $s$, the vector $\varphi(s)$ in eq. 1 can be derived as

$$
\varphi_{i}\left(s_{i}\right)=-q c \sigma_{i}^{-q}\left|s_{i}-\mu_{i}\right|^{q-1} \operatorname{sign}\left(s_{i}-\mu_{i}\right)
$$

Using the GGM-based ICA learning rule (eq. 1), the basis vectors of ship radiated signals and sea noises are extracted respectively. 40,000 samples of each signal were used and the sample-rates were down-sampled to $500 \mathrm{~Hz}$. For each signal, 1000 samples of length $40(8 \mathrm{~ms})$ were generated. Each segment was pre-whitened to improve the convergence speed. The adaptation started from the $40 \times 40$ identity matrix and trained through the 1000 data vectors. The learning rate was gradually decreased from 0.2 to 0.05 during the iteration. When $\mathrm{W}$ is achieved, the basis vectors of signals can be obtained by $\boldsymbol{A}=\boldsymbol{W}^{-1}$. Figure 1 show the basis vectors of ship radiated signals and sea noise.

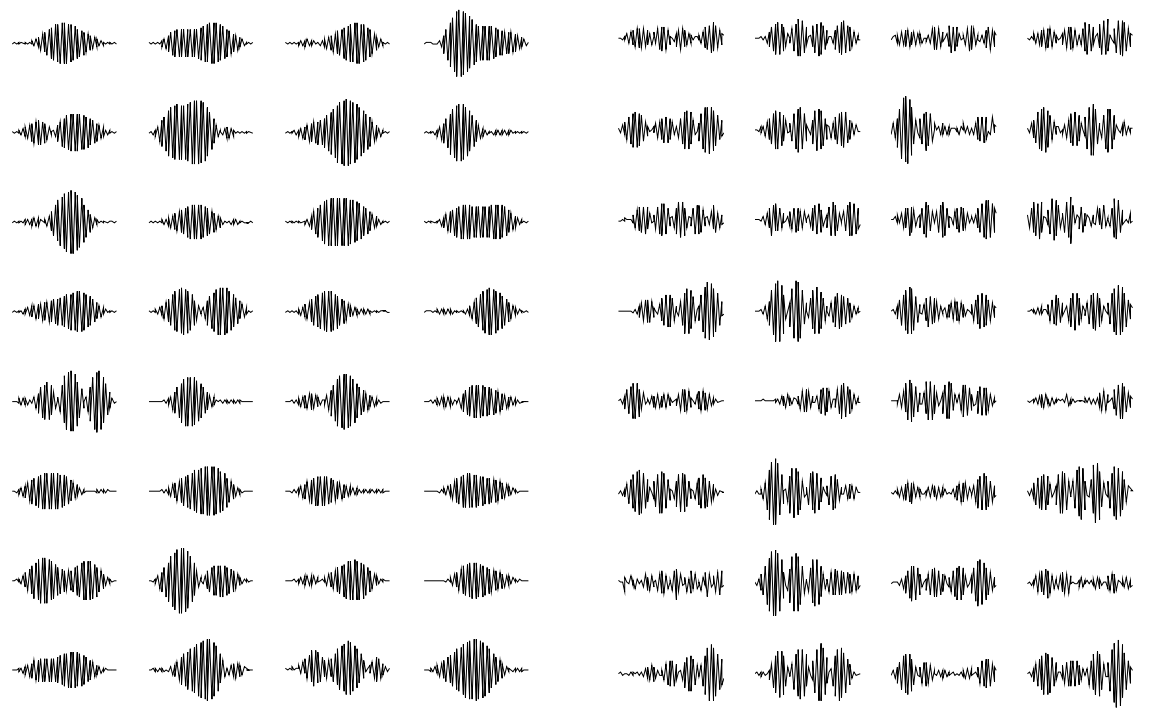

(a)

(b)

Fig. 1. (a)-(b) Basis vectors of ship radiated signals and sea noises in time domain

40 basis vectors of ship radiated signals are show in Fig. 1 (a), in which each subfigure denotes one basis vector which is the column vector of $\boldsymbol{A}$, the same as Fig. 1 (b). The basis vectors look like short-time Fourier bases, but are different in that they are asymmetric in time. The basis vectors of ship radiated signals have one or two peaks and are local in time, but those of sea noises have a few peaks, generally four, and cover all time span like Fourier basis. Fig.2 gives the frequency domain characteristic of fig. 1 . 


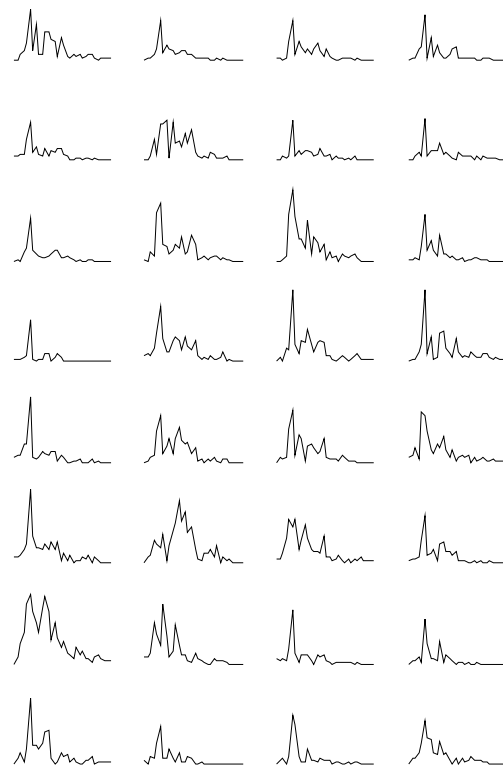

(a)

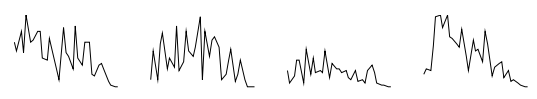

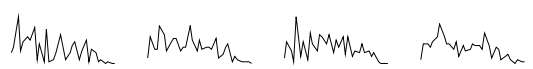

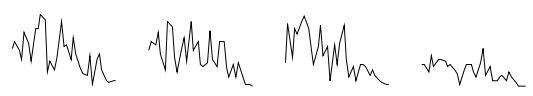

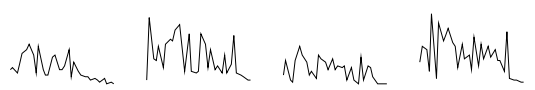

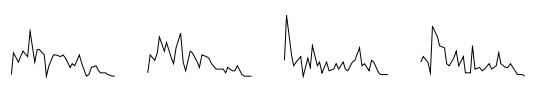

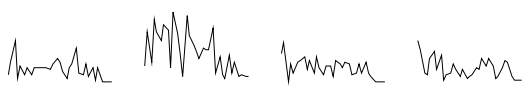

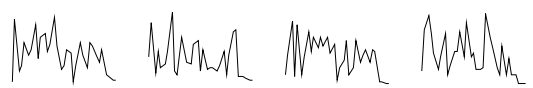

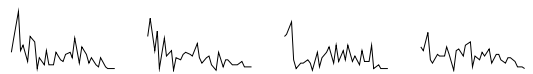

(b)

Fig. 2. (a)-(b) The frequency spectrum of fig.1 (a) and (b)

It can be seen that the ICA basis vectors of ship radiated signals are localized both in time (Fig1. (a)) and frequency domain (Fig2. (a)), and not localized in sea noise (Fig1. (b) and Fig2. (b)). The ICA feature of ship radiated signals are focus on low frequency (Fig.2 (b)), and that of sea noises are global in all frequency domain because sea noises are close to Gaussian distribution.

In order to compare the sparseness of the coefficients produced by ICA and other conventional methods, the log-scaled histograms of the coefficients of DFT, DCT,

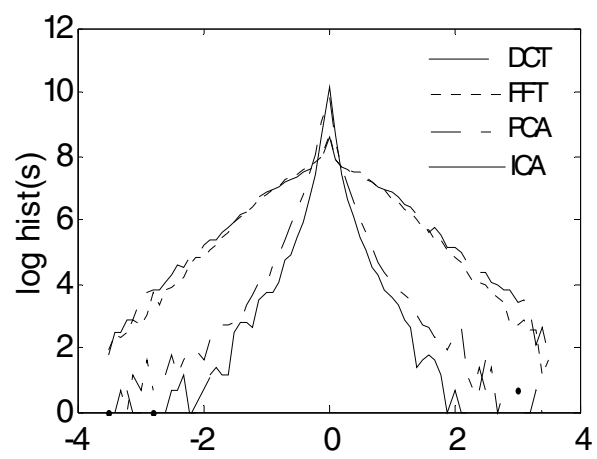

Fig. 3. Histograms of the coefficients of ship radiated signals in different methods 
PCA and ICA for ship radiated signals are shown in fig.3. It can be seen that the distribution of the ICA coefficients is peakier than the others, and this characteristic yields greater sparseness in encode efficiency.

\section{De-noising Method Based on ICA Feature Extraction}

In recently ICA-based de-noising works of speech or image, the de-noising process of noisy signals needs noise-free source data to train the ICA basis vectors as a priori knowledge. Unfortunately, the corresponding noise-free signals are always not acquirable, especially for the underwater acoustic signals. The object ship radiated signals are always submerged in loudly sea noises. In this paper, based on Hyvärinen's maximum likelihood de-noising method [7, 8, 9], an extended method based on GGM-ICA feature extraction is proposed.

In the noise environment, denote $y$ as the noisy coefficient of a basis vector, $s$ as the original noise-free version of coefficient of basis vector, and $v$ as a Gaussian noise with zero mean and variance $\sigma^{2}$. Then the variable $y$ can be describe as

$$
y=s+v
$$

We want to estimate $s$ from the only observed noisy coefficient $y$. Denote $p$ as the probability of $s$, and $f=-\log p$ as its negative $\log$-density, the estimator of $s$ can be obtained by the maximum likelihood (ML) method ${ }^{[7]}$

$$
\hat{s}=\underset{s}{\arg \min } \frac{1}{2 \sigma^{2}}(y-s)^{2}+f(s)
$$

Assuming $f(\cdot)$ to be strictly convex and differentiable, the ML estimation gives the equation

$$
\hat{s}=h(y)
$$

where the nonlinear function $h(\bullet)$ is called as shrinkage function, and the inverse is given by

$$
h^{-1}(s)=s+\sigma^{2} f^{\prime}(s)
$$

Thus, the estimation of $s$ is obtained by inverting a certain function involving $f^{\prime}(\bullet)$.

For current ICA-based de-noising works, however, the de-noising process of noisy signals needs noise-free source data to train the ICA basis vectors. When the corresponding noise-free signals are inaccessible, these algorithms are failed. The GGM-based ICA algorithm in last section has been used to extract the basis vectors directly from noisy signals when the noise-free signals cannot be obtained, and the p.d.f. of the coefficients $p(s)$ learned by the GGM can get simultaneously. Since $f(\bullet)$ in eq. 10 is a function of $p$, the probability of $s$ has been obtained by GGM in ICA feature extraction, so the shrinkage function can be obtained easily.

To recover the de-noised signal from the noisy source three steps are needed. Firstly, by using GGM-based ICA, we can obtain the un-mixing matrix $\boldsymbol{W}$ and the p.d.f. of the corresponding coefficients $p(s)$ at the same time. From the experiments, it 
shows that the coefficients of the basis vectors extracted from noisy underwater acoustic signals have sparse distributions. Secondly, the shrinkage functions can be estimated by $p(s)$ by eq.10, and the de-noised coefficients can be calculated by $\hat{s}=h(y)$. Finally, recover the de-noised ship radiated signals by $\hat{x}=W^{-1} \hat{s}=A \hat{s}$.

This sparse coding method based on ICA may be viewed as a way for determining the basis and corresponding shrinkage functions base on the data themselves. Our method use the transformation based on the statistical properties of the data, whereas the wavelet shrinkage method chooses a predetermined wavelet transform. And the second difference is that we estimate the shrinkage nonlinearities by the ML estimation, again adapting to the data themselves, whereas the wavelet shrinkage method use fixed threshold derived by the min-max principle.

\section{Experiments}

We select 4 kinds of the ship radiated signals mixed with sea noises to test the denoising method. The sampling rate is $500 \mathrm{~Hz}$ and each mixture has 7800 samples. The first step is the feature extraction of the noisy signals using the GGM-based ICA algorithm described in section 2. The un-mixing matrix $\boldsymbol{W}$ was extracted by the learning rule eq. 1 , and it was used as the filter in the de-noising processing. To judge the results of the de-noising, the signal-to-noise ratio (SNR) is used

$$
S N R_{i}=10 \log \left|\frac{\sum_{t=1}^{N} \operatorname{Signal}(t)^{2}}{\sum_{t=1}^{N} \operatorname{Noise}(t)^{2}}\right|
$$

The ship radiated signals mixed with sea noises with the input SNRs of 16.6680 , $6.6346,0.4932$ and -0.6203 respectively. By using the method presented in section 3, we obtain the de-noising results for these 4 kinds of mixtures are 17.7891, 7.7121, 1.9061 and $0.9028 \mathrm{~dB}$ respectively.

To compare the proposed method and conventional de-noising methods, the results of the mean filter $(n=3, n=5)$ and wavelet filter $(d b 3, n=3)$ are also given in table 1 . Where $\mathrm{SNR}_{\text {in }}$ denotes the input SNR of the noisy ship radiated signals and $\mathrm{SNR}_{\text {out }}$ denotes the output SNR of the de-noised signals.

In table 1 , the first column denotes the 4 kinds of mixtures with different input SNR. The second and third columns denotes the de-noising results for these 4 kinds of

Table 1. The de-noising results of 4 kinds of mixtures

\begin{tabular}{|c|c|c|c|c|}
\hline $\begin{array}{c}\mathrm{SNR}_{\text {in }} \text { of noisy } \\
\text { ship radiated } \\
\text { signals }(\mathrm{dB})\end{array}$ & \multicolumn{2}{|c|}{$\begin{array}{c}\mathrm{SNR}_{\text {out }} \text { of mean filter } \\
(\mathrm{dB})\end{array}$} & $\begin{array}{c}\mathrm{SNR}_{\text {out }} \text { of } \\
\text { wavelet filter } \\
(\mathrm{dB})\end{array}$ & $\begin{array}{c}\mathrm{SNR}_{\text {out }} \\
\text { of our } \\
\text { method }(\mathrm{dB})\end{array}$ \\
\cline { 2 - 3 } & $\mathrm{n}=3$ & $\mathrm{n}=5$ & 12.8825 & 17.7891 \\
\hline 16.6680 & 16.3109 & 11.2377 & 12.7121 \\
\hline 6.6346 & 7.1221 & 6.0431 & 7.1446 & 7.7121 \\
\hline 0.4932 & 1.3909 & 1.2900 & 0.9203 & 1.9061 \\
\hline-0.6203 & 0.7743 & 0.7043 & 0.3306 & 0.9028 \\
\hline
\end{tabular}


noisy signals using mean filter with $n=3, n=5$ respectively. The fourth and fifth column are the de-noising results of wavelet filter and our method. For these 4 experiments (each row of the table) we can see that the presented method is efficient and always better than conventional methods. For example, in the second experiment with $\mathrm{SNR}_{\text {in }}=6.6346$, the de-noising result of mean filter and wavelet filter are 7.1221 $(n=3), 6.0431(n=5)$ and 7.7121 respectively, and the results of our method is 7.7121, which is the best in these methods.

Fig. 4 shows the graph of the de-noising results of the second experiment by our method. Fig. 4 (a) is some kind of ship radiated signals, (b) is the sea noises, (c) is the mixture of (a) and (b) with SNR of 6.6346, and (d) is the de-noising result of the mixture. Here we use short-term zero crossing rate (ZCR) to detect the crossing characteristic of the ship. The shortterm ZCR is defined as

$$
Z C R=\frac{1}{2} \sum_{n=1}^{N-1}|\operatorname{sign}[x(n)]-\operatorname{sign}[x(n-1)]|
$$

where $N$ is the number of samples. The short-term ZCR is an efficient method to detect the crossing characteristic of the ship. The short-term ZCR is very high when ship is far away from sonar because the observed signals are almost sea noises which are close to Gaussian distribution in the frequency of $0 \sim 400 \mathrm{~Hz}$. However, when ship comes close to sonar it becomes very low because the ship radiated signals present a strong non-Gaussian distribution in $0 \sim 400 \mathrm{~Hz}$. Fig.5 show the short-term ZCR of fig.4.
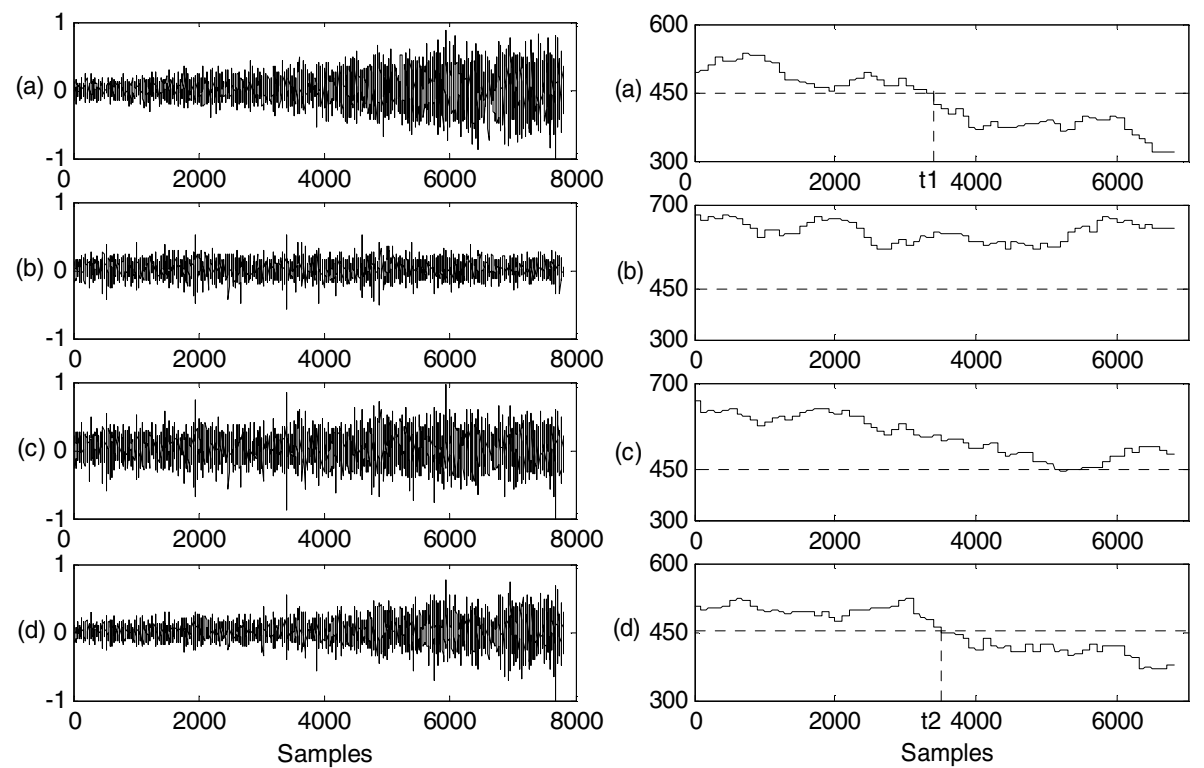

Fig. 4. The de-noising results of noisy ship radiated signals. (a) ship radiated signals, (b) sea noises, (c) the mixture of (a) and (b) with SNR of 6.6346 , (d) the de-noising result of (c).

Fig. 5. (a)-(d) The short-time ZCR of the corresponding signals in fig.4 (a)-(d) 
Fig.5 (a) is the short-term ZCR of the ship radiated signals (Fig.4 (a)), we can see that the value descended quickly after time $t 1=3300 / 500 \mathrm{~Hz}=6.6 \mathrm{~s}$, (b) is the short-term ZCR of sea noise. They are higher than that of ship radiated signals. Therefore, we can choose 450 as the threshold to detect ship signals. (c) is the short-term ZCR of noisy signals. From this figure we can see that the short-term ZCR failed to detect the crossing characteristic of ship since the short-term ZCR higher than the threshold. (4) is the short-term ZCR of de-noised signals. It is clear that the values are lower than the threshold after the time $\mathrm{t} 2=3500 / 500 \mathrm{~Hz}=7 \mathrm{~s}$ which means that the ship can be detected at time $\mathrm{t} 2$.

\section{Conclusions}

Feature extraction and de-noising are important task of pattern recognition of underwater acoustic signals. This paper presented a method of GGM-based ICA feature extraction for underwater acoustic signals. It is demonstrated that the ICA features of underwater acoustic signals are efficient. Since how to extract efficient basis vectors directly from the observed noisy signals is the key objective of noisy signals, in this paper, a method of extracting the basis vectors directly from noisy data is proposed. Sparse coding is achieved by ICA feature extraction in which the ICA features and the shrinkage functions can be obtained simultaneously. By shrinkage the absolute values of the sparse components towards zero, noise can be reduced. Experiments on ship radiated signals mixed with different intensive sea noises show that the proposed method can efficiently remove the additive white Gaussian noise.

\section{References}

1. Te-Won Lee, Gil-Jin Jang, The Statistical Structures of Male and Female Speech Signals, in Proc. ICASSP, (Salt Lack City, Utah), May 2001

2. Jong-Hawn Lee, Ho-Young Jung, Speech Feature Extraction Using Independent Component Analysis, in Proc. ICASP, Istanbul, Turkey, June, 2000,Vol. 3, pp: 1631-1634

3. Anthony J Bell, Terrence J Sejnowski, Learning the Higher-order structure of a nature sound, Network: Computation in Neural System 7 (1996), 261-266

4. Gil-Jin Jang, Te-won Lee, Learning statistically efficient features for speaker recognition, Neurocomputing, 49 (2002): 329-348

5. Miller J. H. \& Thomas J. B., Detectors for Discrete-Time Signals in Non-Gaussian noise, IEEE Transactions on Information Theory, Vol IT-18, no. 2, March 1972. Page(s) 241-250

6. Te-Won Lee, Michael S. Lewicki, The Generalized Gaussian Mixture Model Using ICA, in international workshop on Independent Component Analysis (ICA'00), Helsinki, Finland, June 2000, pp: 239-244

7. Hyvärinen A., Sparse code shrinkage: Denoising of nongaussian data by maximum likelihood estimation. Neural Computation, 1999, 11(7):1739-1768

8. Hyvärinen A., Hoyer P., Oja E., Sparse code shrinkage: Denoising by nonlinear maximum likelihood estimation, Advances in Neural Information Processing System 11 (NIPS'98), 1999

9. Hyvärinen A., Hoyer P., Oja E., Image denoising by sparse code shrinkage, Intelligent Signal Processing, IEEE Press, 2000 